\title{
Meet-in-the-Middle Preimage Attacks on AES Hashing Modes and an Application to Whirlpool
}

\author{
Yu Sasaki \\ NTT Information Sharing Platform Laboratories, NTT Corporation \\ 3-9-11 Midoricho, Musashino-shi, Tokyo 180-8585 Japan \\ sasaki.yu@lab.ntt.co.jp
}

\begin{abstract}
We study the security of AES in the open-key setting by showing an analysis on hash function modes instantiating AES including Davies-Meyer, Matyas-Meyer-Oseas, and Miyaguchi-Preneel modes. In particular, we propose preimage attacks on these constructions, while most of previous work focused their attention on collision attacks or distinguishers using non-ideal differential properties. This research is based on the motivation that we should evaluate classical and important security notions for hash functions and avoid complicated attack models that seem to have little relevance in practice. We apply a recently developed meet-in-the-middle preimage approach. As a result, we obtain a preimage attack on 7 rounds of Davies-Meyer AES and a second preimage attack on 7 rounds of Matyas-Meyer-Oseas and Miyaguchi-Preneel AES. Considering that the previous best collision attack only can work up to 6 rounds, the number of attacked rounds reaches the best in terms of the classical security notions. In our attacks, the key is regarded as a known constant, and the attacks thus can work for any key length in common.
\end{abstract}

Keywords: AES, hash function, Davies-Meyer, Matyas-Meyer-Oseas, Miyaguchi-Preneel, PGV, preimage, meet-in-the-middle, Whirlpool.

\section{Introduction}

Block ciphers are taking important roles in various aspects of our life. Currently, one of the most widely used block-ciphers all over the world is AES [1234.

Since 2009, great progress in the cryptanalysis on AES has been made. Related-key attacks against full-round AES-256 [6 7], full-round AES-192 [6], 7-round AES-128 [9], and 10-round AES-256 with a practical complexity [5] have been proposed. Regarding AES-128, besides the above related-key boomerang attack 9] several non-marginal single-key attacks have been proposed; an impossible differential attack [25] and a single-key attack [15] based on a collision attack [16]. In any attack, the maximum number of attacked rounds is 7 .

On the other hand, block ciphers are sometimes used as hash functions through mode-of-operations. For example, if one needs both a block-cipher and a hash function in a resource-restricted environment such as RFID Tag, only a block-cipher is implemented and a hash function is built using it. Besides, many 
Tag-based applications, such as authentication or anonymity/privacy, do not need the collision resistance [10]. Hence, building a 128-bit hash function with AES is a possible candidate. In fact, [10 proposed 80-bit and 64-bit hash functions using block-cipher PRESENT. Another concern is that many hash functions, even in the SHA-3 competition [35, are designed based on block-ciphers. Hence, block-ciphers' security in hashing modes is an interesting research object.

The known-key attack proposed by Knudsen and Rijmen 21] is the framework for this context. In this model, a secret key is randomly chosen and given to attackers. Then, attackers aim to efficiently detect a certain property of a random instance of the block cipher, where the same property cannot be observed for a random permutation with the same complexity. The attack can be extended to the chosen-key model. e.g. [7. In the known-key model, the key size is irrelevant to the attack. In other words, all key sizes are simultaneously attacked. While, in the chosen-key model, the attack depends on the key-schedule algorithm. Hence, different strategies is necessary for different key sizes.

The first known-key attack was presented by Knudsen and Rijmen 21, which found a non-ideal property of 7-round AES. Then, Mendel et al. presented the known-key attack on 7-round AES [26] based on the rebound attack proposed by Mendel et al. 27. Finally, Gilbert and Peyrin [17] and Lamberger et al. [22] independently applied Super-Sbox analysis to the rebound attack. Gilbert and Peyrin [17] showed that 8-round AES was not ideal in the known-key setting. Regarding the chosen-key attack, Biryukov et al. [7] presented a chosen-key distinguisher on full-round AES-256, which is converted to a $q$-pseudo-collision attack on AES-256 based compression functions. Biryukov and Nikolić also discovered a chosen-key distinguisher on 8-round AES-128 [8].

Although the above results led to significant progress for theoretical cryptanalysis in the secret-, known-, and chosen-key settings, one major drawback is the use of complicated attack models, which are sometimes too theoretic such as related-subkey attacks on block ciphers and distinguishers on block-cipher based compression functions. From this background, several researchers recently have attempted to analyze AES in a simple attack model. For example, Dunkelman et al. [15] and Wei et al. 36] avoided the related-key model and proposed attacks on 8 round AES-256 or AES-192 in the single-key model.

In this paper, we follow the similar principle as Dunkelman et al. [15] and Wei et al. 36. That is to say, we analyze the security of hashing modes instantiating AES in terms of the classical security notions of hash functions, which are actually important for their applications. In particular, we study the preimage resistance of hash functions rather than compression functions.

For hash functions, three security notions are classically considered to be important; collision resistance, second-preimage resistance, and preimage resistance. Among these three, the collision resistance of reduced-round AES can be attacked by applying the techniques used in the rebound attack [27]. In fact, Lamberger et al. [23, Section 5.3] describe a collision attack on an AES-based hash function Whirlpool 30] reduced to 5.5 rounds, which is trivially converted to a collision attack on the Matyas-Meyer-Oseas mode [28, Algorithm 9.41] 
Table 1. Comparison of attacks. 0.5 round of collision and near-collision attacks on Whirlpool by [23] is omitted.

\begin{tabular}{|c|c|c|c|c|c|c|}
\hline Attack & Rounds & Key-size & Mode & $\begin{array}{l}\text { Comp. Func. } \\
\text { (Time, Mem.) }\end{array}$ & $\begin{array}{c}\text { Hash } \\
\text { (Time, Mem.) }\end{array}$ & Ref. \\
\hline \multicolumn{7}{|c|}{ Attacks on AES Hasing modes } \\
\hline Collision & 6 & $128 / 192 / 256$ & MMO,MP & $\left(2^{56}, 2^{32}\right)$ & $\left(2^{56}, 2^{32}\right)$ & 23 \\
\hline 2nd preimage & 6 & $128 / 192 / 256$ & $\mathrm{MMO}, \mathrm{MP}$ & $\left(2^{112}, 2^{16}\right)$ & $\left(2^{112}, 2^{16}\right)$ & Ours \\
\hline 2nd preimage & 7 & $128 / 192 / 256$ & $\mathrm{MMO}, \mathrm{MP}$ & $\left(2^{120}, 2^{8}\right)$ & $\left(2^{120}, 2^{8}\right)$ & Ours \\
\hline Preimage & 6 & $128 / 192 / 256$ & DM & $\left(2^{112}, 2^{16}\right)$ & $\left(2^{121}, 2^{16}\right)$ & Ours \\
\hline Preimage & 7 & $128 / 192 / 256$ & $\mathrm{DM}$ & $\left(2^{120}, 2^{8}\right)$ & $\left(2^{125}, 2^{8}\right)$ & Ours \\
\hline Near col & 7 & $128 / 192 / 256$ & $\mathrm{MMO}, \mathrm{MP}$ & $\left(2^{32}, 2^{32}\right)$ & $\left(2^{32}, 2^{32}\right)$ & 23 \\
\hline Distinguisher & 8 & $128 / 192 / 256$ & $\mathrm{MMO}, \mathrm{MP}$ & $\left(2^{48}, 2^{32}\right)$ & - & 17 \\
\hline$q$-multicollision & 14 & 256 & DM & $\left(q \cdot 2^{67}\right.$, negl. $)$ & - & 7 \\
\hline \multicolumn{7}{|c|}{ Attacks on Whirlpool } \\
\hline Collision & 5 & - & - & $\left(2^{120}, 2^{64}\right)$ & $\left(2^{120}, 2^{64}\right)$ & 23 \\
\hline 2ne Preimage & 5 & - & - & $\left(2^{504}, 2^{8}\right)$ & $\left(2^{504}, 2^{8}\right)$ & Ours \\
\hline Near collision & 7 & - & - & $\left(2^{112}, 2^{64}\right)$ & $\left(2^{112}, 2^{64}\right)$ & 23 \\
\hline Collis & 7 & - & - & $\left(2^{184}, 2^{8}\right)$ & - & 23 \\
\hline Near co & 9 & - & - & $\left(2^{176}, 2^{8}\right)$ & - & 23 \\
\hline Distinguisher & 10 & - & - & $\left(2^{176}, 2^{8}\right)$ & - & 23 \\
\hline
\end{tabular}

instantiating 6-round AES. As far as we know, there is no result that attacks second-preimage resistance or preimage resistance of such an AES usage. Note that the attack by 23] can generate near-collisions on some PGV compression functions with 7-round AES, which might be a valid security notion.

Our Contributions. In this paper, we propose preimage attacks on AES hashing modes including Davies-Meyer (DM) [28, Algorithm 9.42], Matyas-MeyerOseas (MMO), and Miyaguchi-Preneel (MP) [28, Algorithm 9.43] modes. As a result, we obtain a preimage attack on 7 rounds of DM-AES with a complexity of $2^{125} 7$-round AES computations and the memory of $2^{8}$ AES state. We also obtain a second preimage attack on 7 rounds of MMO-AES and MP-AES with a complexity of $2^{120} 7$-round AES computations and the memory of $2^{8}$ AES state. Our attack can also generate second preimages of 5-round Whirlpool with a complexity of $2^{504}$. The attack results are summarized in Table 1 .

We apply a meet-in-the-middle preimage approach developed by Aoki and Sasaki 3. This approach has successfully been applied to many hash functions e.g. MD5 32 and Tiger 18. All of previously analyzed hash functions adopt the DM mode with a relatively weak message schedule, and the weak message schedule is in fact exploited by the attack. However, for AES, the situation is very different because AES has a heavy round function and key schedule. Moreover, it is unclear how to perform preimage attacks against MMO and MP modes.

In our attacks, we fix the value of key-input to a randomly chosen value and search for a plaintext-input that achieves the given hash target. This allows us to attack All PGV modes [29] in the same procedure. We then show that the 
splice-and-cut technique proposed by 3] and the omission of a MixColumns operation in the last round can be combined well and lead to a significant improvement for the preimage attack. Intuitively, this is because the round function without MixColumns is computed as a middle round. This breaks the AES design principle, where AES two rounds achieve the full diffusion, and leads to an attack improvement. Finally, we optimize several techniques of the meet-in-themiddle preimage attack for AES. Specifically, the initial-structure and matching through MixColumns contribute to increase the number of attacked rounds.

Paper Outline. In Sect. 2, we describe AES. In Sect. 3, we introduce previous work. In Sect. 4, we explain a basic idea of our attack. In Sect. 5, we present a preimage attack on 7-round AES. In Sect. 6, we give observations on our attack and apply it to 5-round Whirlpool. Finally, we conclude this paper in Sect. 7 .

\section{Specifications}

Advanced Encryption Standard (AES) 3412 is a 128-bit block cipher supporting three different key sizes; 128, 192, 256 bits. AES computes 10, 12, and 14 rounds for AES-128, -192, and -256, respectively.

By using the key schedule function, round keys are generated from the original secret key. We omit its description because our attacks regard round keys as given constant numbers and thus they are irrelevant to our attacks.

When the data is processed, the internal state is represented by a $4 * 4$ byte array. At the first, the original secret key is XORed to the plaintext, and then, a round function consisting of the following four operations is iteratively applied.

- SubBytes $(S B)$ : substitute each byte according to an S-box table.

- ShiftRows $(S R)$ : apply the $j$-byte left rotation to each byte at row $j$.

- MixColumns $(M C)$ : multiply each column by an MDS matrix. MDS guarantees that the sum of active bytes in the input and output of the MixColumns operation is at least 5 unless all bytes are non-active. The matrices for the encryption and decryption are shown below. Note that $X[j]$ is the input value and $Y[j]$ is the updated value. Numbers with $x_{x}$ are hexadecimal numbers.

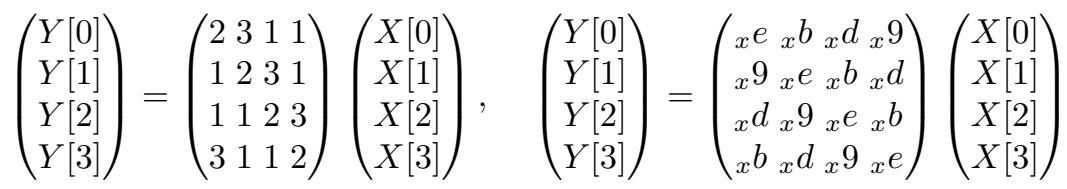

- AddRoundKey $(A K)$ : apply bit-wise exclusive-or with a round key.

Note that the MixColumns operation is not computed at the last round.

Byte positions in a state $S$ are denoted by integer numbers $B, B \in\{0,1,2, \ldots$, $15\}$, as shown in Fig. 11, where the byte $4 j+i$ corresponds to the byte in the $i$-th row and $j$-th column of $S$, and is denoted by $S[4 \cdot j+i]$. We often denote several bytes of state $\mathrm{S}$ by $S[a, b, \ldots]$, e.g. 4 bytes in the right most column are denoted by $S[12,13,14,15]$. 


\begin{tabular}{|c|c|c|c|}
\hline 0 & 4 & 8 & 12 \\
\hline 1 & 5 & 9 & 13 \\
\hline 2 & 6 & 10 & 14 \\
\hline 3 & 7 & 11 & 15 \\
\hline
\end{tabular}

Fig. 1. Byte positions

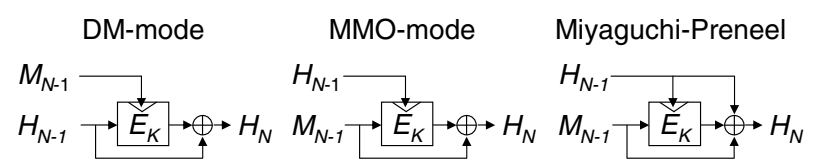

Fig. 2. Illustrations for DM, MMO, and MP modes

Hash Functions Based on Block Ciphers. To build a hash function, we need a domain extension for iteratively applying the compression function. The Merkle-Damgård domain extension is probably mostly used one in practice. It applies the padding to the input message $M$ so that the last block includes the original message length, and splits the padded message to $M_{0}\left\|M_{1}\right\| \cdots \| M_{L-1}$, where the size of each $M_{N}$ is the block length. An initial value $H_{0}$ is defined, and $H_{N}=\mathrm{CF}\left(H_{N-1}, M_{N-1}\right)$ is iteratively applied for $N=1,2, \ldots, L$. Finally, $H_{L}$ is output as a hash value of $M$. This paper assumes that the Merkle-Damgård domain extension is used as a domain extender.

The PGV modes [29] are mode-of-operations to build a compression function from a block cipher. In fact, many hash functions, e.g. MD5, SHA-2, and several SHA-3 candidates, use the PGV modes. Among PGV modes, the DM, MMO, and MP modes are used in practice. Let us denote a block cipher $E$ with a key $K$ by $E_{K}$. The construction of each mode is as follows, which is shown in Fig. 2

$\begin{array}{cl}\text { DM mode: } & \mathrm{CF}\left(H_{N-1}, M_{N-1}\right)=E_{M_{N-1}}\left(H_{N-1}\right) \oplus H_{N-1}, \\ \text { MMO mode: } & \mathrm{CF}\left(H_{N-1}, M_{N-1}\right)=E_{H_{N-1}}\left(M_{N-1}\right) \oplus M_{N-1}, \\ \text { MP mode: } & \mathrm{CF}\left(H_{N-1}, M_{N-1}\right)=E_{H_{N-1}}\left(M_{N-1}\right) \oplus M_{N-1} \oplus H_{N-1} .\end{array}$

\section{Previous Work}

\subsection{Meet-in-the-Middle Preimage Attacks}

To mount the preimage attack, we apply a meet-in-the-middle (MitM) preimage approach developed by Aoki and Sasaki [3, which is based on the pioneering work by Leurent 24] 1 . In this approach, the compression function is divided into two sub-functions so that a portion of bits of the input message only affect one sub-function and another portion of bits of the input message only affect the other sub-function as shown in Fig. 3. This allows attackers to mount the meetin-the-middle attack. Sub-functions are called chunks (stands for chunks of steps or rounds) and bits affecting only one chunk are called neutral. In this paper, we call the chunk that computes the round function in the forward direction forward chunk and in the backward direction backward chunk.

In addition to the basic concept, several techniques have been proposed to extend the attack framework. The splice-and-cut technique 3 regards that the first and last steps are consecutive, and thus any step can be the start point or

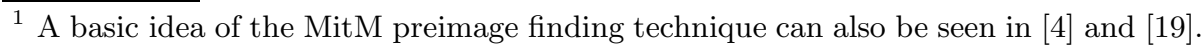




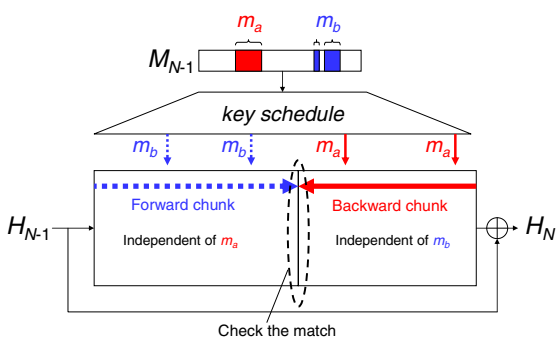

Fig. 3. Basic MitM attack on DM mode

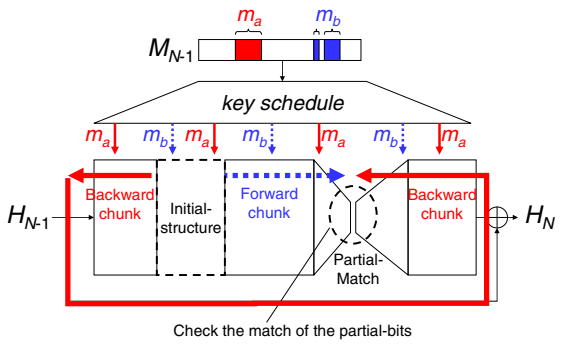

Fig. 4. Advanced techniques for MitM

matching point of the MitM attack. However, as a side-effect, generated items become pseudo-preimages rather than preimages. The local-collision technique [31, initial-structure technique [32] and probabilistic initial-structure technique [18] ignore the order of message words at the start point of the MitM attack. For example in Fig. 4, the order of neutral words $m_{a}$ and $m_{b}$ is reversed between the start points of the forward and backward chunks. These techniques enables MitM attacks even in such a situation. Finally, the partial-matching/-fixing techniques [3] and indirect partial-matching technique [1] match two chunks partially and efficiently. A framework with these techniques is illustrated in Fig. 4.

In $n$-bit narrow-pipe iterated hash functions, pseudo-preimage attacks with a complexity of $2^{m}$, where $m<n-2$, can be converted to preimage attacks with a complexity of $2^{\frac{m+n}{2}+1}$ in generic [28, Fact9.99]. Several researchers showed that pseudo-preimage attacks satisfying certain special properties can be converted to preimage attacks more efficiently than the generic approach [11/1824]. Because our attacks cannot satisfy such properties, we omit their details.

The MitM preimage approach has been applied to many hash functions such as HAVAL 31, MD5 32, reduced SHA-0/-1 [2, reduced SHA-2 1], and Tiger [18. All of previously attacked hash functions adopt the DM mode and their weak key-schedules are exploited by the attack. This strategy cannot work for AES because, in the AES key-schedule, the impact of any change on the secret key or a subkey always propagate to all other subkeys. This indicates that neutral words such as described in Fig. 3 or Fig. 4 do not exist for AES. Moreover, if the message is input as a plaintext in the MMO and MP modes, no input value is available to separate the compression function into two parts.

\subsection{Previous Analysis on AES}

The security of AES in hash function modes was first evaluated by Knudsen and Rijmen [21]. They showed a non-ideal property of 7-round AES. Lamberger et al. showed a collision attack on 5.5-round Whirlpool based on the rebound attack [27, which is trivially converted to a collision attack on 6-round AES. As far as we know, no result is known on the second-preimage or preimage resistance. 
Note that current collision attacks can be applied only if the mode-of-operation is MMO or MP, where attackers fix $H_{N-1}$ and search for $\left(M_{N-1}, M_{N-1}^{\prime}\right)$. If the DM mode is used instead, attackers fix $M_{N-1}$ and search for $\left(H_{N-1}, H_{N-1}^{\prime}\right)$. Hence, only pseudo-collisions on the compression function can be generated.

As analysis methods against the AES block cipher, there exist attacks named collision attack [16] and Meet-in-the-Middle attack [13 (and their extension [15]). These attacks are not related to attacks on hash functions. These attacks based on an observation that a function from a certain input byte to a certain output byte after 4 rounds can be described by 25-byte parameters. Hence, this collision attack does not find paired messages producing an identical state, or this Meet-in-the-Middle attack does not separate the cipher into two independent sub-functions. The goal of these attacks is recovering the secret key of the AES block cipher. Their applicability to hashing modes is not understood well.

\section{Basic Idea of Our Attack and Techniques for Extension}

We first explain a basic idea of our attack by using 4-round AES as an example (Sect.4.1). We fix the block-cipher's key to a constant. This approach is different from previous work in Sect. 3.1 which utilize the independence among subkeys. In this attack, for simplicity, we only apply the splice-and-cut technique. We then explain several techniques to extend the number of attacked rounds (Sect. 4.2).

\subsection{Basic Attack for 4-Round AES}

Goal of the Attack. We fix the key-input when we perform the MitM attack, and the goal is to find the plaintext-input that provides the given target. This approach is irrelevant to the mode-of-operation used. That is, in the DM-mode, we fix a message $M_{N-1}$ to some constant and try to find a chaining variable $H_{N-1}$ that produces the given target $H_{N}$. Similarly, in the MMO- and MP-modes, we fix a chaining variable $H_{N-1}$ and search for a message $M_{N-1}$. Generated pseudo-preimages are later converted to preimages with a technique in Sect. 3.1.

Chunk Separation. We separate 4-round operations into two chunks as shown in Fig. 5] The start point of each chunk is state \#9. We choose \#9[0] as a neutral byte for the forward chunk and \#9[12] for the backward chunk. We fix the other bytes, i.e. $\# 9[1,2, \ldots, 11,13,14,15]$, to randomly chosen values. The backward chunk covers the computation from state \#9 to \#5 and the forward chunk covers from state \#9 to \#16 and \#0 to \#5. Results from two chunks will match at \#5.

Forward Computation. The forward computation starts from \#9. Because $\# 9[12]$ is a neutral byte for the backward chunk, we regard \#9[12] to be unknown during the forward computation. Hence, one byte is unknown at \#11 and the unknown byte is expanded to 4 bytes at \#12. Similarly, by simply tracing the computation, we obtain 8-byte information at \#5 (\#5[0,1,2,3,8,9,10,11]). 


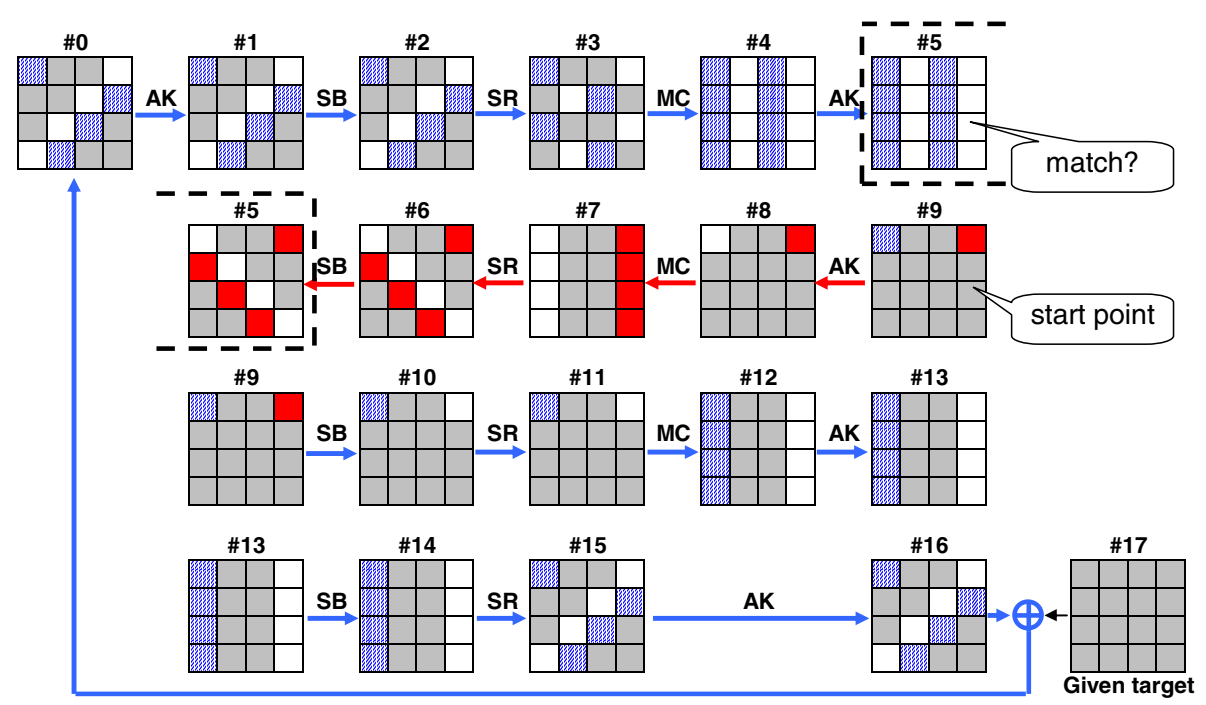

: values depending on neutral bytes for the backward chunk

: values depending on neutral bytes for the forward chunk

: fixed constant

: unknown value

Fig. 5. Basic attack idea: chunk separation for 4-round AES

An important observation is that the omission of the MixColumns operation in the last round extends the number of rounds that can be computed independently. The diffusion of AES is designed so that the full diffusion can be achieved after the 2-round operation. In fact, if MixColumns exists between \#15 and \#16, all bytes become unknown after this operation and it limits the attack efficiency strongly. However, the omission of MixColumns yields 12 known bytes at \#16, and to make things worse, the positions of unknown bytes will overlap by the next ShiftRows operation, and thus attackers can compute MixColumns for another round. As a conclusion, we can summarize this property as follows;

AES 2-rounds achieve the full diffusion, however, if MixColumns is omitted in the second round, 4 rounds are needed to achieve the full diffusion.

This property is illustrated in Fig. 6. This situation does not seem to occur for the AES block cipher. However, in hash function modes, the splice-and-cut can exploit it by starting the forward chunk from the second last round.

Overall Attack Procedure. Because the backward computation is similar and straight-forward, we omit its explanation. Overall, if we fix 14 bytes at \#9 as shown in Fig. 5], we can compute the forward chunk for $2^{8}$ values of $\# 9[0]$ and obtain 8-byte information at \#5. The obtained results are stored in a table and sorted. Similarly, we can compute the backward chunk for $2^{8}$ values of \#9[12] and obtain 12-byte information at \#5. Because 2 bytes $(\# 5[1,11])$ are overlapped 

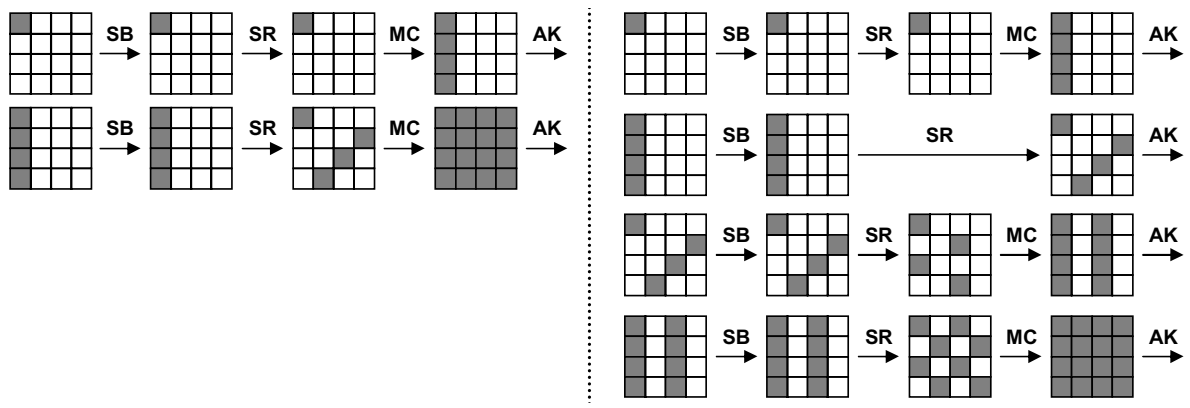

Fig. 6. Slow diffusion with the omission of MixColumns in the second round

between the results from both chunks, we can efficiently check the match of those results. If the match is not found, the attack is repeated by changing the values of fixed 14 bytes at \#9 or the values for the AES key-input.

Each chunk can be built using $2^{8}$ possible neutral values with $2^{8}$ computations. The results are stored in a table of the size $2^{8}$ AES state and then sorted. After that, $2^{16}$ pairs are tested in the 2-byte match and 1 pair will succeed. Hence, if we repeat the attack $2^{112}$ times, we will find a pair that also matches other 14 bytes. The final complexity for generating pseudo-preimages is $2^{8} \cdot 2^{112}=2^{120}$. This is converted to a preimage attack on the hash function with a complexity of $2^{\frac{120+128}{2}+1}=2^{125}$ using a generic conversion in Sect. 3.1.

Note that the attack efficiency is not optimized because the purpose of this attack is to demonstrate the basic idea of our attack. Also note that, the impact of the change of \#9[12] does not propagate to all bytes at the matching stage. This is because the backward chunk is too short. If the number of attacked rounds is extended as explained in Sect. 5, the impact will propagate to all bytes.

\subsection{Techniques for Attacking More Rounds}

We show that a technique similar to the initial-structure 32 can extend the number of rounds in each chunk by one round (in total 2 rounds), and by considering the MixColumns operation deeply, we can include one more round during the matching stage. These techniques are directly applied to our 7-round attack that will be explained in Sect. 5. Specifically, the differential path described in Fig. 7 and Fig. 8 are the copy of a part of differential path in Fig. 9 .

Initial-Structure. The idea is choosing several bytes as neutral bytes, and determining these values so that several output bytes of the MixColumns or InverseMixColumns operations can be constant values. This minimizes the number of unknown bytes after the first MixColumns operation in each chunk, and thus, the number of attacked rounds is extended by one round in each chunk. The construction of the initial-structure is shown in Fig. 7. 


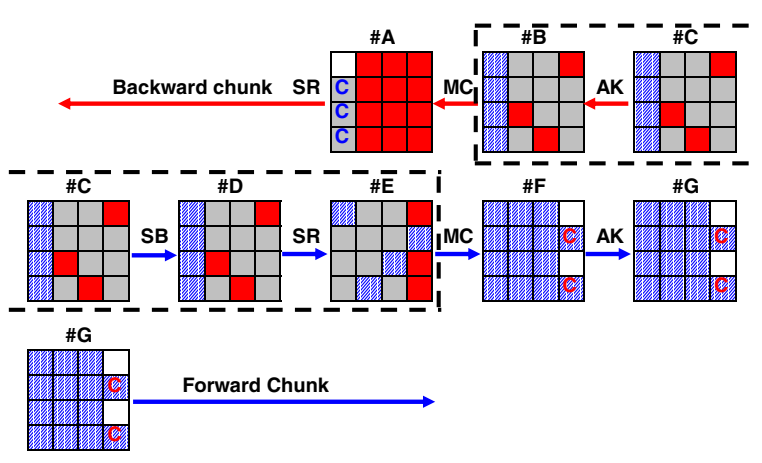

Fig. 7. Initial-structure technique for AES

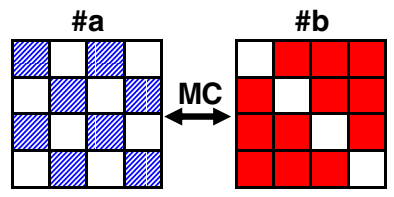

Fig. 8. Known-byte patterns for matching through MixColumns

The neutral bytes for the forward chunk are 4 bytes \#B[0,1,2,3]. We choose $2^{8}$ values of these bytes and use them to compute the forward chunk independently of the backward chunk. These values are determined as follows;

1. Randomly choose constant values for 3 bytes at \#A (\#A[1,2,3]).

2. For all possible $2^{8}$ values of $\# \mathrm{~B}[0]$, we calculate the values of $\# \mathrm{~B}[1,2,3]$ so that the chosen 3 bytes at \#A $(\# \mathrm{~A}[1,2,3])$ can be achieved through the InverseMixColumns operation. Note that, according to Eq. (11), \#A[1,2,3] is computed by Eq.(2). Because there are 3 free variables to control 3 bytes, this is possible by solving a system of equations.

$$
\left(\begin{array}{l}
\# A[1] \\
\# A[2] \\
\# A[3]
\end{array}\right)=\left(\begin{array}{l}
{ }_{x} 9{ }_{x} e{ }_{x} b{ }_{x} d \\
{ }_{x} d{ }_{x} 9{ }_{x} e{ }_{x} b \\
{ }_{x} b{ }_{x} d{ }_{x} 9{ }_{x} e
\end{array}\right)\left(\begin{array}{l}
\# B[0] \\
\# B[1] \\
\# B[2] \\
\# B[3]
\end{array}\right)
$$

As a result, for any $2^{8}$ neutral values of \#B[0,1,2,3], \#A[1,2,3] become constant, and thus, the backward computation from \#A can start with 15 known bytes and only 1 unknown byte.

The neutral bytes for the backward chunk are 3 bytes \#E[12,14,15]. We choose $2^{8}$ values of these bytes as follows;

1. Randomly choose constant values for 2 bytes, which will be the impact from \#E[12,14,15] to the chosen 2 bytes at \#F (\#F[13,15]). In details, by considering the MixColumns operation in Eq. (1), $\# \mathrm{~F}[13,15]$ are written as follows:

$$
\begin{aligned}
& \# F[13]=(1 \cdot \# E[12]) \oplus(2 \cdot \# E[13]) \oplus(3 \cdot \# E[14]) \oplus(1 \cdot \# E[15]), \\
& \# F[15]=(3 \cdot \# E[12]) \oplus(1 \cdot \# E[13]) \oplus(1 \cdot \# E[14]) \oplus(2 \cdot \# E[15]) .
\end{aligned}
$$


The impacts on $\# \mathrm{~F}[13]$ and $\# \mathrm{~F}[15]$ from \# $\mathrm{E}[12,14,15]$ mean the following values respectively.

$$
\begin{aligned}
& (1 \cdot \# E[12]) \oplus(3 \cdot \# E[14]) \oplus(1 \cdot \# E[15]), \\
& (3 \cdot \# E[12]) \oplus(1 \cdot \# E[14]) \oplus(2 \cdot \# E[15]) .
\end{aligned}
$$

2. For all possible $2^{8}$ values of \#E[12], we calculate the values of \#E[14,15] by solving a system of equations so that the impact on the chosen 2 bytes at \#F $(\# \mathrm{~F}[13,15])$ can be achieved through the MixColumns operation. Because there are 2 free variables to control 2 bytes, this is always possible.

As a result, for any $2^{8}$ neutral values of $\# \mathrm{E}[12,14,15]$, the impact from these values to $\# \mathrm{~F}[13,15]$ becomes the determined constant. Note that $\# \mathrm{~F}[13,15]$ are also influenced by \#E[13], and thus, final values of $\# \mathrm{~F}[13,15]$ are exclusive-or of the determined constant and values depending on \#E[13]. Finally, the forward computation from \#F can start with 14 known bytes and only 2 unknown bytes.

Match through MixColumns. Assume that many values of the partially known states of the form \#a and \#b in Fig. 8 are stored in tables. The goal of this match is efficiently finding paired values $(\# \mathrm{a}, \# \mathrm{~b})$ that match through the MixColumns operation. Because MixColumns is applied column by column, the match is also tested column by column. We explain the match for the first column as an example. The other columns can be tested in the same procedure.

Let us consider the InverseMixColumns operation from \#b to \#a. From Eq. (1) , \#a[0] and \#a[2] are expressed as follows;

$$
\begin{aligned}
& \# a[0]=\left({ }_{x} e \cdot \# b[0]\right) \oplus\left({ }_{x} b \cdot \# b[1]\right) \oplus\left({ }_{x} d \cdot \# b[2]\right) \oplus\left({ }_{x} 9 \cdot \# b[3]\right) \\
& \# a[2]=\left({ }_{x} d \cdot \# b[0]\right) \oplus\left({ }_{x} 9 \cdot \# b[1]\right) \oplus\left({ }_{x} e \cdot \# b[2]\right) \oplus\left({ }_{x} b \cdot \# b[3]\right)
\end{aligned}
$$

Considering that $\# \mathrm{~b}[1,2,3]$ are known values, the equations can be transformed by using some constant numbers $C_{0}$ and $C_{1}$ as follows;

$$
\# a[0] \oplus C_{0}={ }_{x} e \cdot \# b[0], \quad \# a[2] \oplus C_{1}={ }_{x} d \cdot \# b[0] .
$$

Whether or not these equations are satisfied can be checked efficiently by using the idea based on the indirect partial-matching [1]. Namely, ${ }_{x} d \cdot\left(\# a[0] \oplus C_{0}\right)=$ ${ }_{x} e \cdot\left(\# a[2] \oplus C_{1}\right)$ is obtained from Eq. (9), and then obtain the following equation:

$$
{ }_{x} d \cdot \# a[0] \oplus{ }_{x} e \cdot \# a[2]={ }_{x} d \cdot C_{0} \oplus{ }_{x} e \cdot C_{1} .
$$

Let us denote ${ }_{x} d \cdot \# a[0] \oplus{ }_{x} e \cdot \# a[2]$ and ${ }_{x} d \cdot C_{0} \oplus{ }_{x} e \cdot C_{1}$ by $C_{\text {for }}$ and $C_{b a c k}$, respectively. By computing $C_{\text {for }}$ and $C_{b a c k}$ in the computation for each chunk, we can perform the match by just comparing these values.

Note that AES has 4 columns in a state. Because the number of candidates for the match can be reduced by a factor of $2^{-8}$ per column, for 4 columns, the number of candidates is reduced by a factor of $2^{-32}$. 


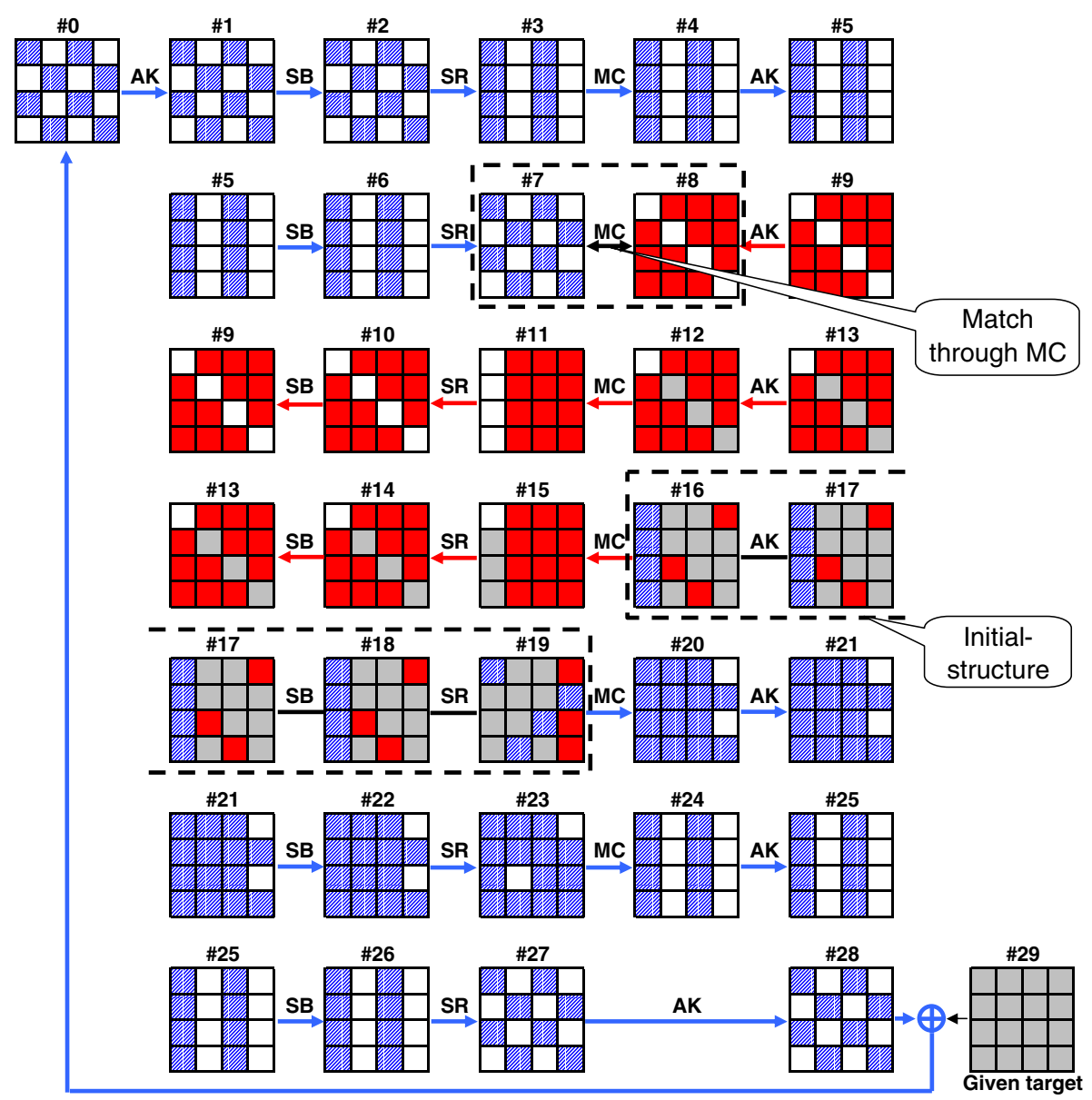

Fig. 9. Chunk separation for 7-round AES

\section{Preimage Attack against 7-Round AES}

By considering the techniques explained in Sect. 4.2, we can attack up to 7 rounds of AES. The chunk separation for this attack is depicted in Fig. 9.

In this attack, states \#16 to \#19 are chosen as the initial-structure and we apply the match between states \#7 and \#8. The neutral bytes for the forward computation are 4 bytes at \#16, namely, \#16[0,1,2,3]. The neutral bytes for the backward computation are 3 bytes at \#19, namely, \#19[12,14,15].

To make the initial-structure work, we choose neutral bytes for the forward chunk so that 3 bytes \#15[1,2,3] can be pre-determined constant values. Similarly, neutral bytes for the backward chunk are computed so that impacts on 2 bytes \#20[13,15] can be pre-determined constant values. The matching procedure is exactly the same as the one explained in Section 4.2. The detailed attack 
procedure is explained below. Note that the procedure below is a preimage attack on the compression function. To convert this attack to the one for a hash function, we need additional effort depending on the mode-of-operation used.

1. Choose a value for the key-input, and compute all sub-keys. How to choose the value depends on the mode-of-operation. In this procedure, we assume that the key-input can be any value. We remove this assumption later.

2. Randomly choose constant values for 9 bytes in state \#16 (\#16[4,5,7,8,9,10, $13,14,15])$, for 3 bytes in state \#15 (\#15[1,2,3]), and for impacts on 2 bytes in state \#20 (\#20[13,15]) from the neutral bytes of the backward chunk.

3 . For all $2^{8}$ values of \#16[0], do as follows.

(a) Calculate $\# 16[1,2,3]$ so that 3 bytes $\# 15[1,2,3]$ can be pre-determined constant values.

(b) Compute the forward chunk from \#20 to \#28, and then, from \#0 to \#7. Also compute $C_{\text {for }}$ in Eq. (10) for each column.

(c) Store the results in a table $T_{\text {for }}$.

4. Sort the table $T_{\text {for }}$ after obtaining $2^{8}$ values.

5. For all $2^{8}$ values of \#19[12], do as follows.

(a) Calculate \#19[14,15] so that the impacts from these values to 2 bytes \#20[13,15] can be pre-determined constant values.

(b) Compute the backward chunk from \#15 to \#8.

(c) From 12 known bytes of \#8, compute $C_{\text {back }}$ in Eq. (10) for each column.

(d) Check if there exists an entry in $T_{\text {for }}$ that matches the computed $C_{\text {back }}$.

(e) If exits, compute all bytes of $\# 7$ and $\# 8$ with matched values and check if all 128-bit values of \#7 and \#8 match.

(f) If all 128-bits match, output the corresponding $\left(H_{N-1}, M_{N-1}\right)$.

6. If the attack does not succeed with $2^{8}$ values of \#19[12], go back to Step 2 (or Step 1 if necessary) and repeat the attack with different constant values.

Complexity Evaluation. In this attack, the sum of the complexity for computing $2^{8}$ results of $\# 7$ and $\# 8$ is roughly $2^{8} 7$-round AES computations. At Step $3 \mathrm{c}$, we need a memory to store $2^{8} \cdot 4$-byte information for $C_{f o r}$. At Step $5 \mathrm{~d}$ we search for the table of the size $2^{8}$. Hence, for all $2^{8}$ values of \#19[12], the cost is about $2^{8} \cdot \log 2^{8}$ memory access, which is enough small compared to $2^{8} 7$-round AES for computing \#7 and \#8. The success probability of the match is $2^{-8}$ for each column, and thus $2^{-32}$ for 4 columns. Hence, after generating $2^{8}$ results for $\# 7$ and $2^{8}$ results for $\# 8,2^{8} \cdot 2^{8} \cdot 2^{-32}=2^{-16}$ candidate will remain, where a remaining candidate satisfies 4 -byte linear relations in a state. Therefore, if we iterate the above procedure $2^{112}$ times, we obtain $2^{112} \cdot 2^{-16}=2^{96}$ candidates satisfying the match and one of them will satisfy the other 12-byte linear relations in a state, in other words, a preimage on the compression function is found. Note that, at Step 6, the algorithm can go back to Step 2 up to $2^{112}$ times for a fixed key-input. To repeat the attack more, we need to change the key-input at Step 11. The final complexity of the attack is $2^{8} \cdot 2^{112}=2^{120}$ AES 7-round computations and we need a memory for storing $2^{8} \cdot 4$-byte information. 


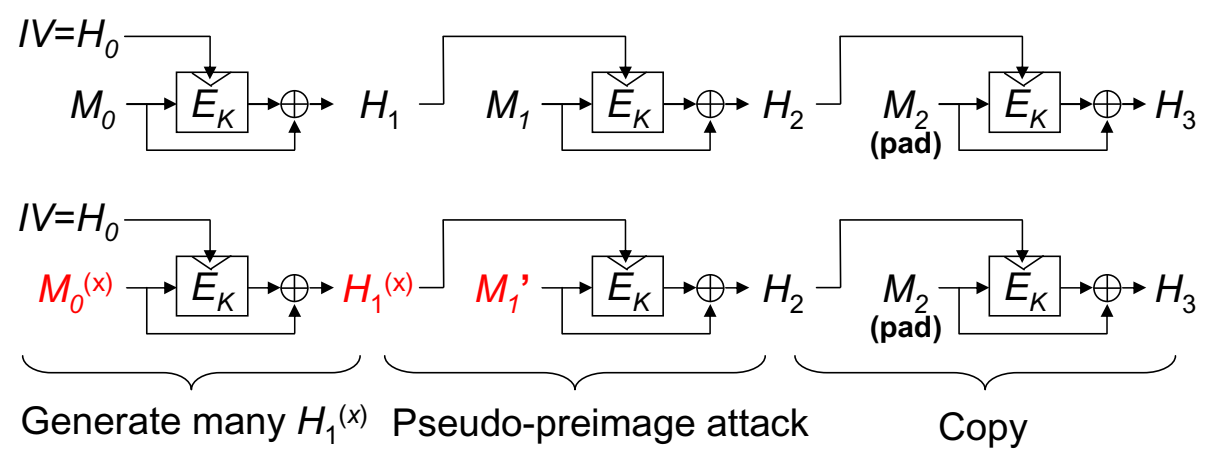

Fig. 10. (Top) Given first-preimage (Bottom) Second-preimage construction

Conversion to Hash Function Scenario. How to convert this attack to the hash function scenario depends on the mode-of-operation used. For the DM mode, this attack finds $\left(H_{N-1}, M_{N-1}\right)$, where the value of $M_{N-1}$ is chosen at Step 1 and the value of $H_{N-1}$ is determined randomly during the attack. In this scenario, we can choose the message so that the padding string can be satisfied. Instead, $H_{N-1}$ cannot be fixed to IV. Hence, we choose $M_{N-1}$ at Step 1 so that the padding string for 2-block messages is satisfied, and convert pseudopreimages into preimages with the conversion in Sect. 3.1. Finally, the attack generates preimages of 2 -block long with a complexity of $2^{1+(120+128) / 2}=2^{125}$.

For the MMO or MP modes, the value of $H_{N-1}$ is chosen at Step 1 and the value of $M_{N-1}$ is determined randomly during the attack. Therefore, we can always start from the IV, but cannot satisfy the padding string. Because of the padding problem, this attack cannot generate preimages. Hence, we aim to generate second preimages. Assume that the given first preimage is 3-block long. The attack is depicted in Fig. 10. Our attack copies the last message block in which the padding string is included. For the first block, we choose several different message values $M_{0}^{(x)}$ to find several different chaining variables $H_{1}^{(x)}$. Finally, for the second block, we choose one of generated $H_{1}^{(x)}$ and search for a message $M_{1}^{\prime}$ that satisfies $\mathrm{CF}\left(M_{1}^{\prime}, H_{1}^{(x)}\right)=H_{2}$ using the pseudo-preimage attack. Hence, second preimages are generated with a complexity of $2^{120}$. Note that, a fixed $H_{2}$ cannot be mapped from a fixed $H_{1}^{(x)}$ for any $M_{1}^{\prime}$ with a probability of $1-e^{-1}$. In such a case, we choose another $H_{1}^{(x)}$ and repeat the attack. After several trials of $H_{1}^{(x)}$, we will find a valid $M_{1}^{\prime}$ with a probability almost 1 .

\section{Discussion}

Other PGV Modes. In PGV, 12 schemes in Table 2 are secure. Our attacks can be applied to all 12 schemes. In our attack, the key is chosen and fixed. Therefore, as long as the key is equivalent to $H_{i}$, the same attack as the 
Table 2. Twelve secure PGV constructions. $X_{i}$ represents $H_{i} \oplus M_{i}$.

\begin{tabular}{|c|c||c|c||c|c||c|c|}
\hline No. & Computation & No. & Computation & No. & Computation & No. & Computation \\
\hline 1 & $E_{H_{i}}\left(M_{i}\right) \oplus M_{i}$ & 2 & $E_{H_{i}}\left(X_{i}\right) \oplus X_{i}$ & 3 & $E_{H_{i}}\left(M_{i}\right) \oplus X_{i}$ & 4 & $E_{H_{i}}\left(X_{i}\right) \oplus M_{i}$ \\
\hline 5 & $E_{M_{i}}\left(H_{i}\right) \oplus H_{i}$ & 6 & $E_{M_{i}}\left(X_{i}\right) \oplus X_{i}$ & 7 & $E_{M_{i}}\left(H_{i}\right) \oplus X_{i}$ & 8 & $E_{M_{i}}\left(X_{i}\right) \oplus H_{i}$ \\
\hline 9 & $E_{X_{i}}\left(M_{i}\right) \oplus M_{i}$ & 10 & $E_{X_{i}}\left(H_{i}\right) \oplus H_{i}$ & 11 & $E_{X_{i}}\left(M_{i}\right) \oplus H_{i}$ & 12 & $E_{X_{i}}\left(H_{i}\right) \oplus M_{i}$ \\
\hline
\end{tabular}

MMO-mode can be performed. Hence, on No.1 to No.4, a second-preimage attack with $2^{120}$ computations is possible. Similarly, on No.5 to No.8, a preimage attack with $2^{125}$ computations is possible. On No.9 to No.12, the key $X_{i}$ is chosen but either $H_{i}$ or $M_{i}$ cannot be chosen. Hence, only a second-preimage attack with $2^{125}$ computations is possible. Considering the long message attack [20], our attack has an advantage only if the length of the given message is 3- to 7-blocks.

As a further generalization, applications to the generalized PGV construction proposed by [33] seems interesting. We leave this work as an open problem.

Complexity on AES 6-Rounds. To demonstrate the change of the complexity with a different number of rounds, we attacked 6-rounds. The idea is omitting the match through the $M C$ and apply the direct match instead. This enables attackers to reduce the number of known bytes in the backward chunk, and thus to keep $2^{16}$ neutral values for each chunk. The final results are listed in Table 1 .

Known-Key Attack on 7-Round AES. Our attack can be regarded as a new approach of the known-key attack on AES, which finds fixed points on 7-round AES. The success probability of the attack is $1-e^{-1}$.

The attacker is given a randomly chosen key $k$. Then, she carries out the pseudo-preimage attack on 7-round AES in Sect. 5 with setting the given target hash value to 0 . With a complexity of $2^{120}$, a plaintext $p$ s.t. $p=E_{k}(p)$ will be found, while finding such $p$ will cost $2^{128}$ for a 128 -bit random permutation.

Note that Gilbert and Peyrin proposed a known-key distinguisher on 8-round AES [17. They find some non-ideal differential property, while our attack finds a fixed point which has been discussed for a long time. The application of our known-key distinguisher to the hash function scenario is meaningful, which finds a preimage of 0 in several PGV constructions. However, [17] attacks 8-round with a feasible complexity, while ours attacks 7-round with an infeasible complexity.

Difficulties in Chosen-Key Setting. In our attack, the key-input is fixed to a constant. It might be possible to extend the attack by actively choosing the keyinput. However, this is not trivial. Firstly, the splice-and-cut technique cannot be used for the key-schedule function, namely, most part of the first round key cannot be obtained from the last round key without computing the inversion. Hence, the MitM attack would be difficult. Secondly, the previous related-key attacks on AES focused their attention on differential properties. It is unclear how to use the weak property of the key-schedule function to build preimages. 


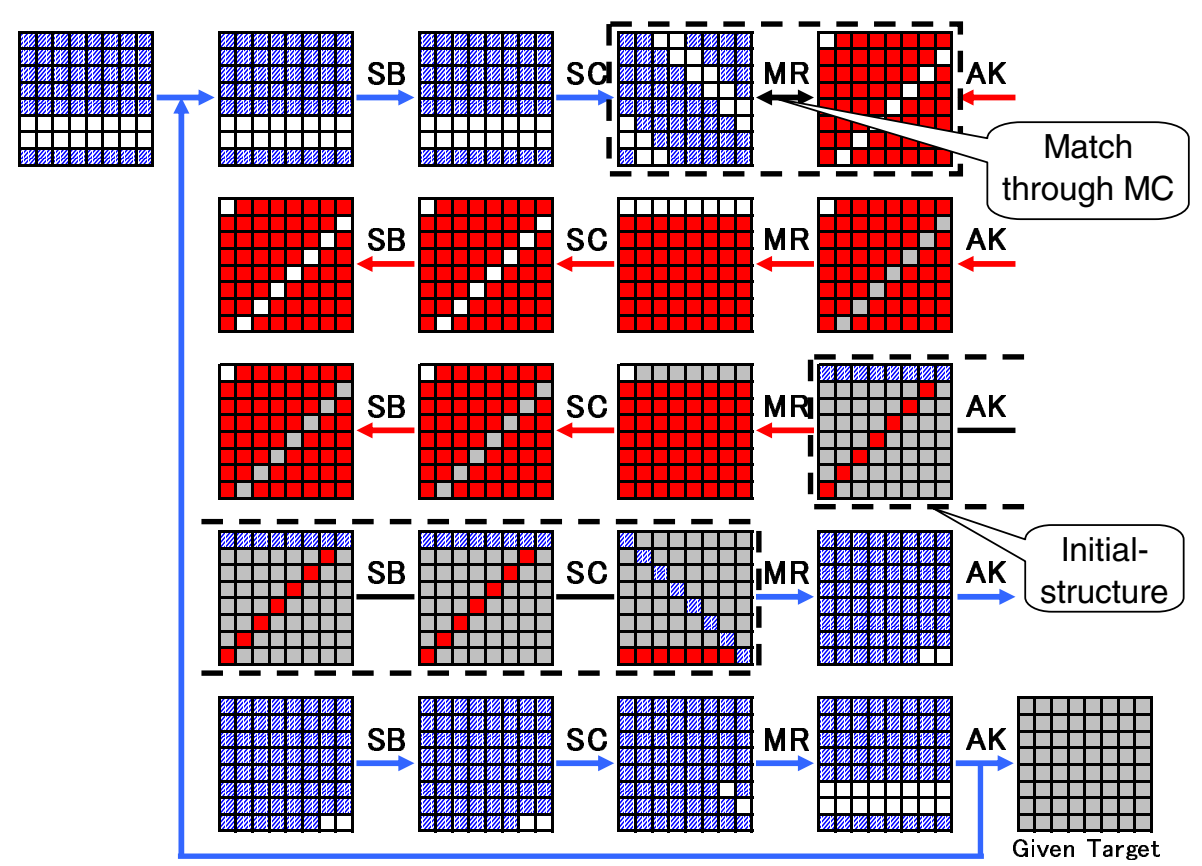

Fig. 11. Chunk separation for second preimage attack on 5-round Whirlpool. Whirlpool computes ShiftColumns(SC) and MixRows(MR) instead of SR and MC.

Application for Whirlpool. Our attack exploits the omission of MixColumns in the last round. Here, we discuss a variant of AES, which computes MixColumns in the last round2. To check the number of attacked rounds, we again use Fig. 9. Due to MixColumns in the last round, we need to remove the 7 th round from our target. Note that known byte positions at states \#25 and \#5 are identical. This indicates that we also need to remove the first round, and state \#25 should be connected to state \#6. As a result, we can attack only 5 rounds.

Whirlpool [30] is a hash function which is deeply based on AES with a larger state size. Because Whirlpool computes MixColumns during the last round, the above analysis can be directly applied as a preimage attack on reduced Whirlpool. Whirlpool uses $8 * 8$-byte (512-bit) state and consists of 10 naturallyexpanded AES rounds. It produces the compression function output with the MP mode. In Fig. 11, we show the chunk separation for attacking 5-round Whirlpool.

The attack strategy is the same as 7-round attack on AES. Hence we omit the details. Pseudo-preimages can be generated faster than the brute force attack by a factor of $2^{8}$, which is $2^{504}$. Because Whirlpool uses the MP mode, this can be a second-preimage attack with a complexity of $2^{504}$ and a memory of $2^{8}$.

${ }^{2}$ One may note another study on the effects of the omission of MixColumns [14]. 


\section{Concluding Remarks}

In this paper, we studied the security of AES hashing modes in terms of the classical and important security notions. We proposed a preimage attack on the PGV modes instantiating AES by applying the meet-in-the-middle approach. As a result, we obtained a preimage attack on 7 rounds of DM-AES and secondpreimage attack on 7 rounds of MMO-AES and MP-AES. This attack can also generate second preimages of Whirlpool reduced to 5 rounds.

Note that our results do not give impact on other AES-based hash functions e.g. several SHA-3 candidates, in particular, those with the wide-pipe structure.

\section{Acknowledgements}

I would like to thank the anonymous reviewers of FSE 2011 for many fruitful comments, especially for the research motivation and applications of the attack.

\section{References}

1. Aoki, K., Guo, J., Matusiewicz, K., Sasaki, Y., Wang, L.: Preimages for StepReduced SHA-2. In: Matsui, M. (ed.) ASIACRYPT 2009. LNCS, vol. 5912, pp. 578-597. Springer, Heidelberg (2009)

2. Aoki, K., Sasaki, Y.: Meet-in-the-middle preimage attacks against reduced SHA-0 and SHA-1. In: Halevi, S. (ed.) CRYPTO 2009. LNCS, vol. 5677, pp. 70-89. Springer, Heidelberg (2009)

3. Aoki, K., Sasaki, Y.: Preimage attacks on one-block MD4, 63-step MD5 and more. In: Avanzi, R.M., Keliher, L., Sica, F. (eds.) SAC 2008. LNCS, vol. 5381, pp. 103-119. Springer, Heidelberg (2009)

4. Aumasson, J.-P., Meier, W., Mendel, F.: Preimage Attacks on 3-Pass HAVAL and Step-Reduced MD5. In: Avanzi, R.M., Keliher, L., Sica, F. (eds.) SAC 2008. LNCS, vol. 5381, pp. 120-135. Springer, Heidelberg (2009)

5. Biryukov, A., Dunkelman, O., Keller, N., Khovratovich, D., Shamir, A.: Key recovery attacks of practical complexity on AES-256 variants with up to 10 rounds. In: Gilbert, H. (ed.) EUROCRYPT 2010. LNCS, vol. 6110, pp. 299-319. Springer, Heidelberg (2010)

6. Biryukov, A., Khovratovich, D.: Related-key cryptanalysis of the full AES-192 and AES-256. In: Matsui, M. (ed.) ASIACRYPT 2009. LNCS, vol. 5912, pp. 1-18. Springer, Heidelberg (2009)

7. Biryukov, A., Khovratovich, D., Nikolić, I.: Distinguisher and related-key attack on the full AES-256. In: Halevi, S. (ed.) CRYPTO 2009. LNCS, vol. 5677, pp. 231-249. Springer, Heidelberg (2009)

8. Biryukov, A., Nikolić, I.: A new security analysis of AES-128. In: Rump Session of CRYPTO 2009 (2009), http://rump2009.cr.yp.to/

9. Biryukov, A., Nikolić, I.: Automatic search for related-key differential characteristics in byte-oriented block ciphers: Application to AES, camellia, khazad and others. In: Gilbert, H. (ed.) EUROCRYPT 2010. LNCS, vol. 6110, pp. 322-344. Springer, Heidelberg (2010) 
10. Bogdanov, A., Leander, G., Paar, C., Poschmann, A., Robshaw, M.J.B., Seurin, Y.: Hash functions and RFID tags: Mind the gap. In: Oswald, E., Rohatgi, P. (eds.) CHES 2008. LNCS, vol. 5154, pp. 283-299. Springer, Heidelberg (2008)

11. De Cannière, C., Rechberger, C.: Preimages for reduced SHA-0 and SHA-1. In: Wagner, D. (ed.) CRYPTO 2008. LNCS, vol. 5157, pp. 179-202. Springer, Heidelberg (2008)

12. Daemen, J., Rijmen, V.: The design of Rijndeal: AES - the Advanced Encryption Standard (AES). Springer, Heidelberg (2002)

13. Demirci, H., Selçuk, A.A.: A meet-in-the-middle attack on 8-round AES. In: Nyberg, K. (ed.) FSE 2008. LNCS, vol. 5086, pp. 116-126. Springer, Heidelberg (2008)

14. Dunkelman, O., Keller, N.: The effects of the omission of last round's MixColumns on AES. Cryptology ePrint Archive, Report 2010/041 (2010), http://eprint. iacr. org/2010/041

15. Dunkelman, O., Keller, N., Shamir, A.: Improved single-key attacks on 8-round AES-192 and AES-256. In: Abe, M. (ed.) ASIACRYPT 2010. LNCS, vol. 6477, pp. 158-176. Springer, Heidelberg (2010)

16. Gilbert, H., Minier, M.: A collision attack on 7 rounds Rijndael. In: Third AES Candidate Conference (AES3), pp. 230-241. Springer, Heidelberg (2000)

17. Gilbert, H., Peyrin, T.: Super-sbox cryptanalysis: Improved attacks for AES-like permutations. In: Hong, S., Iwata, T. (eds.) FSE 2010. LNCS, vol. 6147, pp. 365383. Springer, Heidelberg (2010)

18. Guo, J., Ling, S., Rechberger, C., Wang, H.: Advanced meet-in-the-middle preimage attacks: First results on full tiger, and improved results on MD4 and SHA-2. In: Abe, M. (ed.) ASIACRYPT 2010. LNCS, vol. 6477, pp. 56-75. Springer, Heidelberg (2010)

19. Indesteege, S., Preneel, B.: Preimages for reduced-round tiger. In: Lucks, S., Sadeghi, A.-R., Wolf, C. (eds.) WEWoRC 2007. LNCS, vol. 4945, pp. 90-99. Springer, Heidelberg (2008)

20. Kelsey, J., Schneier, B.: Second preimages on $n$-bit hash functions for much less than $2^{n}$ work. In: Cramer, R. (ed.) EUROCRYPT 2005. LNCS, vol. 3494, pp. 474-490. Springer, Heidelberg (2005)

21. Knudsen, L.R., Rijmen, V.: Known-key distinguishers for some block ciphers. In: Kurosawa, K. (ed.) ASIACRYPT 2007. LNCS, vol. 4833, pp. 315-324. Springer, Heidelberg (2007)

22. Lamberger, M., Mendel, F., Rechberger, C., Rijmen, V., Schläffer, M.: Rebound distinguishers: Results on the full whirlpool compression function. In: Matsui, M. (ed.) ASIACRYPT 2009. LNCS, vol. 5912, pp. 126-143. Springer, Heidelberg (2009)

23. Lamberger, M., Mendel, F., Rechberger, C., Rijmen, V., Schläffer, M.: The rebound attack and subspace distinguishers: Application to Whirlpool. Cryptology ePrint Archive, Report 2010/198 (2010), http://eprint.iacr.org/2010/198

24. Leurent, G.: MD4 is not one-way. In: Nyberg, K. (ed.) FSE 2008. LNCS, vol. 5086, pp. 412-428. Springer, Heidelberg (2008)

25. Lu, J., Dunkelman, O., Keller, N., Kim, J.-S.: New impossible differential attacks on AES. In: Chowdhury, D.R., Rijmen, V., Das, A. (eds.) INDOCRYPT 2008. LNCS, vol. 5365, pp. 279-293. Springer, Heidelberg (2008)

26. Mendel, F., Peyrin, T., Rechberger, C., Schläffer, M.: Improved cryptanalysis of the reduced gr $\varnothing$ stl compression function, ECHO permutation and AES block cipher. In: Jacobson Jr., M.J., Rijmen, V., Safavi-Naini, R. (eds.) SAC 2009. LNCS, vol. 5867, pp. 16-35. Springer, Heidelberg (2009) 
27. Mendel, F., Rechberger, C., Schläffer, M., Thomsen, S.S.: The rebound attack: Cryptanalysis of reduced whirlpool and gr $\varnothing$ stl. In: Dunkelman, O. (ed.) FSE 2009. LNCS, vol. 5665, pp. 260-276. Springer, Heidelberg (2009)

28. Menezes, A.J., van Oorschot, P.C., Vanstone, S.A.: Handbook of Applied Cryptography. CRC Press, Boca Raton (1997)

29. Preneel, B., Govaerts, R., Vandewalle, J.: Hash functions based on block ciphers: A synthetic approach. In: Stinson, D.R. (ed.) CRYPTO 1993. LNCS, vol. 773, pp. 368-378. Springer, Heidelberg (1994)

30. Rijmen, V., Barreto, P.S.L.M.: The WHIRLPOOL hashing function. Submitted to NISSIE (September 2000)

31. Sasaki, Y., Aoki, K.: Preimage attacks on 3, 4, and 5-pass HAVAL. In: Pieprzyk, J. (ed.) ASIACRYPT 2008. LNCS, vol. 5350, pp. 253-271. Springer, Heidelberg (2008)

32. Sasaki, Y., Aoki, K.: Finding preimages in full MD5 faster than exhaustive search. In: Joux, A. (ed.) EUROCRYPT 2009. LNCS, vol. 5479, pp. 134-152. Springer, Heidelberg (2009)

33. Stam, M.: Blockcipher-based hashing revisited. In: Dunkelman, O. (ed.) FSE 2009. LNCS, vol. 5665, pp. 67-83. Springer, Heidelberg (2009)

34. U.S. Department of Commerce, National Institute of Standards and Technology. Specification for the ADVANCED ENCRYPTION STANDARD (AES) (Federal Information Processing Standards Publication 197) (2001), http://csrc.nist. gov/encryption/aes/index.html\#fips

35. U.S. Department of Commerce, National Institute of Standards and Technology. Federal Register /Vol. 72, No. 212/Friday, November 2, 2007/Notices (2007), http://csrc.nist.gov/groups/ST/hash/documents/FR_Notice_Nov07.pdf

36. Wei, Y., Lu, J., Hu, Y.: Meet-in-the-middle attack on 8 rounds of AES block cipher under 192 key bits. Cryptology ePrint Archive, Report 2010/537 (2010), http:// eprint.iacr.org/2010/537 (appeared in the accepted papers list of ISPEC 2011) 